\title{
HYDROMECHANICAL RESPONSES OF A DECOMMISSIONED BACKFILLED TUNNEL DRILLED INTO A PORO-VISCOELASTIC MEDIUM
}

\author{
N. Dufour ${ }^{\mathrm{i})}$, C. J. Leo ${ }^{\mathrm{ii})}$, F. Deleruyelle ${ }^{\mathrm{iii}}$ and H. Wong ${ }^{\mathrm{i}}$
}

\begin{abstract}
This paper is an extension of earlier papers (Wong et al., 2008a, b) which sought to investigate the poroelastic and poro-viscoelastic behaviour of a ground medium following the closing of a spherical cavity, whereas the focus here is for a long horizontal tunnel with a circular section. It presents Laplace transform analytic solutions for the time dependent hydromechanical responses of a decommissioned and backfilled deep tunnel drilled into a poro-viscoelastic host medium. Both the backfill, which is assumed as poroelastic, and the host medium are supposed saturated with water. The solutions developed and inverted numerically to obtain the responses in time domain are used to study the effects of lining support deterioration, the backfill stiffness and of the host medium viscosity. The convergence of the tunnel leads to the expulsion of water from the backfill into the host medium resulting in corresponding transient responses. Each of the key parameters studied can have the effect of either attenuating or accentuating the transient and long term hydromechanical responses by delaying or shortening the dissipation period depending on the direction of change taken by the parameter value. The solutions presented in this paper permit an assessment of the relative importance of these parameters. Comparisons are also drawn between a tunnel and a spherical cavity of identical radius. Generally speaking, the implications of lining support, backfill stiffness and viscosity of ground medium are more significant in terms of the resulting hydromechanical responses, for the tunnel than for the spherical cavity.
\end{abstract}

Key words: creep, hydromechanical, Laplace transform, numerical inversion, poro-viscoelastic, tunnel convergence, tunnel lining (IGC: E13/G5/G13/H5)

\section{INTRODUCTION}

One of the major concerns that faces the nuclear industry is to find a solution for the disposal of high-level longlived radioactive waste. There is an increasing international consensus that deep geological repository would be the preferred solution, and this approach has been the focus of much research interests in recent years. Figure 1 (Andra, 2005) shows a typical layout of an underground disposal facility imaginable in a clay geological formation. It is made of a deep network of tunnels. At the end of the service life of the underground facility, it is anticipated that the tunnels will be backfilled and closed off. Depending on the properties of the surrounding ground, the lining support of the tunnels may be left in place. For purpose of long term safety analysis of the disposal facility, ground water movements, which may lead to early dissemination of radioactive isotopes, must be assessed. This paper focuses on the influence of the backfill stiffness and of the deterioration rate of the lining support on the hydromechanical responses of a creeping surrounding ground long after the underground facility has been closed off. A rigorous analysis of this task would require

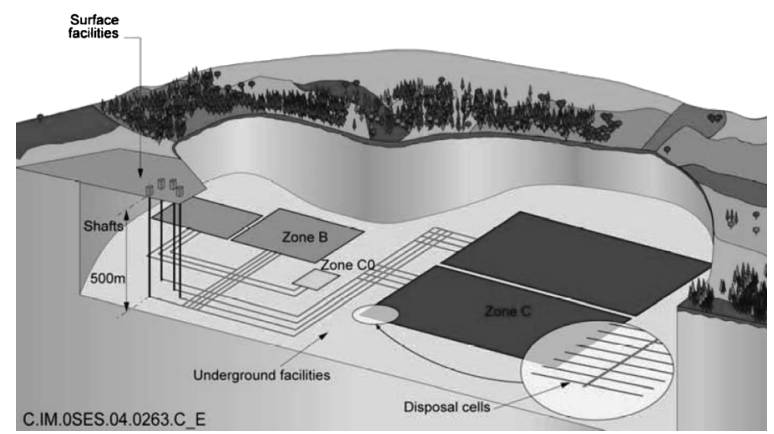

Fig. 1. Typical layout of an underground disposal facility imaginable in a clay geological formation (from Andra, 2005)

complex numerical simulations coupling poromechanical and viscoplastic behaviour of geomaterials. However, analytical solutions are very useful as a tool for orders of magnitude analyses and for parametric studies aiming at better understanding the influence of changes in characteristics of system components. They also provide very useful benchmark tests to validate complex numerical calculations. However, such analytical solutions are also

i) Université de Lyon, France.

ii) School of Engineering, University of Western Sydney, NSW 1797, Australia (c.leo@uw.edu.au).

iii) Institut de Radioprotection et de Sûreté Nucléaire, France.

The manuscript for this paper was received for review on May 9, 2008; approved on March 26, 2009.

Written discussions on this paper should be submitted before March 1, 2010 to the Japanese Geotechnical Society, 4-38-2, Sengoku, Bunkyo-

$\mathrm{ku}$, Tokyo 112-0011, Japan. Upon request the closing date may be extended one month. 
quite rare in the open literature. Thus, any new analytical solutions are of added value.

In the present paper we have developed some quasianalytical solutions for the purpose of studying the influence of the backfill stiffness and the lining deterioration of a long circular section tunnel on the hydromechanical evolution of the surrounding ground. The solutions take into account viscoelastic creep coupled to water flow, and are "quasi-analytical" in nature since they are derived in the Laplace transform space and require numerical inversion to obtain the real solutions in time domain. The tunnel is considered to be backfilled with a poroelastic material, and both ground and backfill are supposed saturated with fluid (water). The timedependent creep behaviour of the host medium after the tunnel closing is simulated using a poro-viscoelastic model. In the context of tunneling, creep is widely regarded as a key mechanism causing increase in ground movement and support loading (e.g., Phienwej et al., 2007; Phienwej, 1987; Semple, 1973; Terzaghi, 1946), hence the inclusion of viscous effects in the host medium model is important. Stability is another critical issue but it has been studied elsewhere (e.g., Subrin and Wong, 2002). The present work for a circular tunnel is an extension of our earlier papers (Wong et al., 2008a, b) which sought to investigate the poroelastic and poro-viscoelastic behaviour of the surrounding ground as a result of the closing of a spherical cavity.

\section{THE BACKFILLED CIRCULAR TUNNEL PROBLEM}

\section{Constitutive Equations of a Poro-viscoelastic Medium}

Before proceeding further and for the sake of subsequent mathematical developments, we will now introduce the Laplace transform, defined for a typical function $f(r, t)$ as follows:

$$
\bar{f}(r, s)=L\{f(r, t)\}=\int_{0}^{\infty} f(r, t) e^{-\mathrm{st}} d t
$$

and the inverse as,

$$
f(r, t)=L^{-1}\{\bar{f}(r, s)\}=\frac{1}{2 \pi i} \int_{\Gamma-i \infty}^{\Gamma+i \infty} \bar{f}(r, s) e^{s t} d s
$$

where $s$ is the Laplace transform parameter and $i^{2}=-1$. In the notations adopted in the present paper, the bar over the symbol denotes the transformed function represented by the symbol. The value $\Gamma$ is chosen such that all poles in the $s$-plane lie to the left of the vertical line $\operatorname{Re}(\mathrm{s})=\Gamma$. As will be observed below, Laplace transform equations will be used interchangeably with their real time counterparts during the development process.

In classical poroelasticity, the properties of the porous structure of the medium are presupposed to be independent of time effects. This is a satisfactory assumption in many instances provided the time effects are small and can be ignored. In the framework of the present study, the ground movements and increases of the support loading are caused by the creep of the host rock and thus, must be taken into account. In light of this and to include the possibility of creep effects, the behaviour of the porous structure of the surrounding medium of the tunnel, can be described by the following poro-viscoelastic functional constitutive equations (e.g., Coussy, 1991, 2004; Wong et al., 2008a; Dufour, 2008):

$$
\begin{aligned}
& \delta \sigma+\delta p=K \hat{\otimes} \epsilon ; \quad \delta \bar{\sigma}+\delta \bar{p}=s \bar{K} \bar{\epsilon} \\
& \delta s_{\mathrm{ij}}=2 \mu \hat{\otimes} e_{\mathrm{ij}} ; \quad \delta \overline{\mathrm{ij}}_{\mathrm{ij}}=2 s \bar{\mu} \bar{e}_{\mathrm{ij}} \\
& \epsilon=K^{-1} \hat{\otimes}(\delta \sigma+\delta p) ; \quad \bar{\epsilon}=s \overline{K^{-1}}(\delta \bar{\sigma}+\delta \bar{p}) \\
& e_{\mathrm{ij}}=\frac{1}{2} \mu^{-1} \hat{\otimes} \delta s_{\mathrm{ij}} ; \quad \bar{e}_{\mathrm{ij}}=\frac{1}{2} s \overline{\mu^{-1}} \delta \bar{s}_{\mathrm{ij}}
\end{aligned}
$$

where

$$
a \hat{\otimes} \mathrm{b}=\int_{0}^{t} a(t-\tau) \frac{\partial b(\tau)}{\partial \tau} d \tau+\sum_{i} a\left(t-t_{\mathrm{i}}\right)\left[b\left(t_{\mathrm{i}}^{+}\right)-b\left(t_{\mathrm{i}}^{-}\right)\right]
$$

defines the Stieltjes convolution integral involving the functions $a(t)$ and $b(t)$, taking into account the discontinuities of the function $b$ at time $t_{\mathrm{i}} ; K, \mu$ are the drained bulk and shear relaxation kernels; $K^{-1}, \mu^{-1}$ are the drained bulk and shear creep kernels; $\sigma=\sigma_{\mathrm{kk}} / 3$ is the mean stress and $s_{\mathrm{ij}}=\sigma_{\mathrm{ij}}-\delta \sigma_{\mathrm{ij}}$ is the deviatoric stress tensor; $\epsilon=\varepsilon_{\mathrm{kk}}$ is the volumetric strain and $e_{\mathrm{ij}}=\varepsilon_{\mathrm{ij}}-(\epsilon / 3) \delta_{\mathrm{ij}}$ is the deviatoric strain tensor; $\delta$ denotes the increment with respect to the initial state, e.g., $\delta \sigma=\sigma-\sigma^{0}$ gives the total stress increment above initial stress $\sigma^{0}$ while $\delta p=p-p_{0}$ denotes the excess pore pressure above $p_{0}$. The kernels $K$ and $\mu$ are given functions of time for modeling the timedependent response of the host material, in this case the surrounding ground. Generally, these functions belong to one of the following 3 categories (Findley et al., 1976):

(a) an empirical mathematical curve fitting to a given set of data;

(b) the mathematical description of a relatively simple, abstract physical model composed of various combinations of mechanical elements such as springs and dashpots;

(c) a rigorous theoretical treatment deriving from observed physical and assumed rheological behaviour of a given material.

The well known power law (a specialised form of Findley's equation) exemplifies an empirical model (category (a)), whereas the Kelvin and Maxwell models constitute examples of the rheological mechanical types (category (b)). Models derived on the basis of micro mechanics approach where the material behaviour is investigated at two different scale levels, the micro-scale and the macroscale levels, would fall in the last category (c). We adopt the following well known $K, \mu$ functions:

$$
\begin{aligned}
& K(t)=\left\{K_{\infty}+\left(K_{0}-K_{\infty}\right) e^{-t / \tau_{\mathrm{r}}}\right\} H(t) ; \\
& \bar{K}(s)=\frac{K_{0}}{s}\left\{\frac{K_{\infty}}{K_{0}}+\frac{s\left(1-K_{\infty} / K_{0}\right)}{\left(s+1 / \tau_{\mathrm{r}}\right)}\right\} \\
& \mu(t)=\left\{\mu_{\infty}+\left(\mu_{0}-\mu_{\infty}\right) e^{-t / \theta_{\mathrm{r}}}\right\} H(t) ; \\
& \bar{\mu}(s)=\frac{\mu_{0}}{s}\left\{\frac{\mu_{\infty}}{\mu_{0}}+\frac{s\left(1-\mu_{\infty} / \mu_{0}\right)}{\left(s+1 / \theta_{\mathrm{r}}\right)}\right\}
\end{aligned}
$$

where $H(t)$ is the Heaviside function, $K_{\infty}, \mu_{\infty}$ are the 


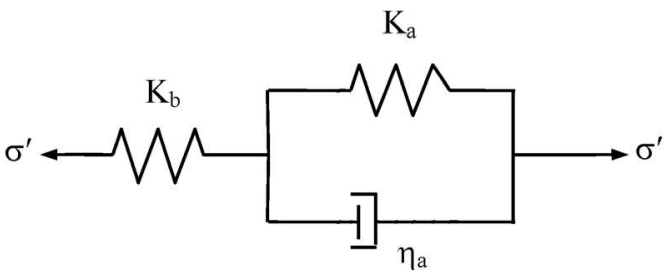

(a)

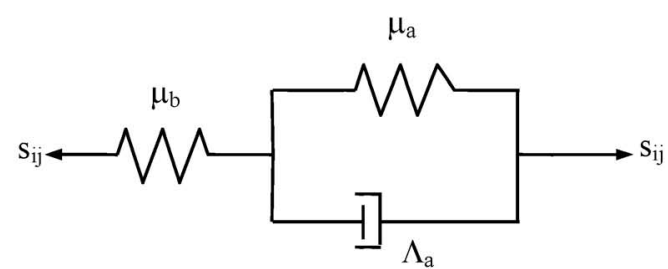

(b)

Fig. 2. Kevin-Voigt models for (a) bulk and (b) shear visco-elastic behaviour

drained long term (delayed) bulk and shear moduli; $K_{0}, \mu_{0}$ are the drained short term (instantaneous) bulk and shear moduli; $\tau_{\mathrm{r}}, \theta_{\mathrm{r}}$ are the characteristic relaxation times of the volumetric and shear strains respectively. The "short" or "long" term notions spoken about in this paper must be understood in relation to the values of $\tau_{\mathrm{r}}$ and $\theta_{\mathrm{r}}$ parameters. The use of Eqs. (7) and (8) with the short and long term moduli to simulate creep effects is not only a choice of simplicity for the purpose of solving analytically the equations, it is also widely adopted in engineering practice to simulate time dependent geomaterials. Equations (7) and (8) are, in fact, special forms of the KelvinVoigt rheological model (Fig. 2) where equivalence is established if the following is chosen:

$$
\begin{aligned}
& K_{\mathrm{b}}=K_{0} ; \quad K_{\mathrm{a}}=\frac{K_{0} K_{\infty}}{K_{0}-K_{\infty}} ; \quad \eta_{\mathrm{a}}=\tau_{\mathrm{r}} K_{\mathrm{a}} \\
& \mu_{\mathrm{b}}=\mu_{0} ; \quad \mu_{\mathrm{a}}=\frac{\mu_{0} \mu_{\infty}}{\mu_{0}-\mu_{\infty}} ; \quad \Lambda_{\mathrm{a}}=\theta_{\mathrm{r}} \mu_{\mathrm{a}}
\end{aligned}
$$

We further note that even though Eqs. (7) and (8) are adopted in the present study, there is no loss of generality in the general Laplace transformed solutions developed below as they can be applied for any general functions of $K, \mu$ as long as they are Laplace transformable and invertible. For completeness, we can also invoke the classic identities of the Stieltjes convolution integral to invert Eqs. (7) and (8) to give the bulk and shear compliances:

$$
\begin{aligned}
& K^{-1}(t)=\left\{\frac{1}{K_{\infty}}+\left(\frac{1}{K_{0}}-\frac{1}{K_{\infty}}\right) e^{-\mathrm{t} / \tau_{\mathrm{c}}}\right\} H(t) ; \\
& \overline{K^{-1}}(s)=\frac{1}{s K_{0}}\left\{\frac{K_{0}}{K_{\infty}}+\frac{s\left(1-K_{0} / K_{\infty}\right)}{\left(s+1 / \tau_{\mathrm{c}}\right)}\right\} \\
& \mu^{-1}(t)=\left\{\frac{1}{\mu_{\infty}}+\left(\frac{1}{\mu_{0}}-\frac{1}{\mu_{\infty}}\right) e^{-\mathrm{t} / \theta_{\mathrm{c}}}\right\} H(t) ; \\
& \overline{\mu^{-1}}(s)=\frac{1}{s \mu_{0}}\left\{\frac{\mu_{0}}{\mu_{\infty}}+\frac{s\left(1-\mu_{0} / \mu_{\infty}\right)}{\left(s+1 / \theta_{\mathrm{c}}\right)}\right\}
\end{aligned}
$$

where $\tau_{\mathrm{c}}, \theta_{\mathrm{c}}$ are the characteristic creep times which are related to the above relaxation times as follows (e.g., Wong et al., 2008a; Dufour, 2008):

$$
\frac{\tau_{\mathrm{r}}}{\tau_{\mathrm{c}}}=\frac{K_{\infty}}{K_{0}} ; \quad \frac{\theta_{\mathrm{r}}}{\theta_{\mathrm{c}}}=\frac{\mu_{\infty}}{\mu_{0}}
$$

Note that Eqs. (3)-(6) transform into the classical constitutive equations of poroelasticity when the functions are independent of time.

\section{Governing Equations}

The quasi-static equations of equilibrium for the ground medium, initially at $t=0$ and subsequently at $t>0$ are given by:

$$
\operatorname{div}\left(\sigma_{\mathrm{ij}}^{0}\right)+b_{\mathrm{i}}^{0}=0 ; \quad \operatorname{div}\left(\sigma_{\mathrm{ij}}\right)+b_{\mathrm{i}}=0
$$

where $b_{\mathrm{i}}^{0}, b_{\mathrm{i}}$ are the body forces, typically consisting of the self weight of the medium, that is: $\mathbf{b}^{0}=\left(0,0,-\rho_{0} g\right)^{T}$, $\mathbf{b}=(0,0,-\rho g)^{T} ; \rho_{0}=\left(1-\phi_{0}\right) \rho_{\mathrm{s}}+\phi_{0} \rho_{\mathrm{f}}$ and $\rho=(1-\phi) \rho_{\mathrm{s}}+$ $\phi \rho_{\mathrm{f}} ; \rho_{\mathrm{f}}, \rho_{\mathrm{s}}, \rho, \rho_{0}$ are the fluid, skeleton, bulk and the initial densities respectively; $\phi, \phi_{0}$ are the current and initial porosity of the medium. However, in the absence of occluded porosity, it can be assumed that the fluid and the solid grains of the skeleton of the porous medium generally undergo negligible volume changes. Therefore, the constituents can be considered as incompressible such that the fluid density, $\rho_{\mathrm{f}}$, and the skeleton density, $\rho_{\mathrm{s}}$, remain constant in time. Moreover, we will suppose that the variation of unit weight due to porosity change is negligible so that the difference of the first and the second equation in Eq. (14) will yield:

$$
\operatorname{div}\left(\delta \sigma_{\mathrm{ij}}\right)=0
$$

Since $\delta \sigma_{\mathrm{ij}}=\delta \sigma \delta_{\mathrm{ij}}+\delta s_{\mathrm{ij}}$, then making use of Eqs. (3) and (4) and substituting into Eq. (15) gives:

$$
\operatorname{div}\left\{(s \bar{K} \bar{\epsilon}-\delta \bar{p}) \delta_{\mathrm{ij}}+2 s \bar{\mu} \bar{e}_{\mathrm{ij}}\right\}=0
$$

The displacement field of the surrounding ground $\mathbf{u}(r, t)$ in the presence of an axially symmetrical tunnel is irrotational, thus $\operatorname{rot}(\operatorname{rot}(\mathbf{u}))=\mathbf{0}$, so that $\operatorname{div}(\varepsilon)=\operatorname{grad}(\epsilon)$ and $\operatorname{div}(\mathbf{e})=2 / 3 \operatorname{grad}(\epsilon)$ and we get from Eq. (16):

$$
\operatorname{grad}\{\omega(s) \bar{\epsilon}-\delta \bar{p}\}=\mathbf{0} ; \quad \omega(s)=s\left(\bar{K}+\frac{4}{3} \bar{\mu}\right)
$$

In a saturated porous medium, the transport of the fluid within the pores and its interaction with the solid skeleton must also be considered. Consequently, from the consideration of mass balance of the fluid mass in the pores of the medium (e.g., Coussy, 2004; Pereira, 2005), we write:

$$
\frac{\partial \phi}{\partial t}+\operatorname{div}\left(\frac{\mathbf{w}_{\mathrm{f}}}{\rho_{\mathrm{f}}}\right)=0
$$

where $\mathbf{w}_{\mathrm{f}}\left(=\rho_{\mathrm{f}} \phi\left(\mathbf{V}_{\mathrm{f}}-\mathbf{V}_{\mathrm{s}}\right)\right)$ is the fluid mass flux, while $\mathbf{V}_{\mathrm{f}}$, $\mathbf{V}_{\mathrm{s}}$ are the velocities of the pore fluid and of the grains of the medium skeleton respectively. Since the pore fluid is initially in static equilibrium, Darcy's Law gives, 


$$
\frac{\mathbf{w}_{\mathrm{f}}}{\rho_{\mathrm{f}}}=-\lambda_{\mathrm{h}} \operatorname{grad}(\delta p)
$$

where $\lambda_{\mathrm{h}}\left(=k_{\mathrm{h}} / \gamma_{\mathrm{w}}\right)$ is the permeability; $k_{\mathrm{h}}$ is the hydraulic conductivity of the medium and $\gamma_{\mathrm{w}}$ is the unit weight of the fluid. Assuming infinitesimal strain, that is: $\|\nabla \mathbf{u}\| \ll 1$, and since the solid grains constituting the medium are considered incompressible we get $\epsilon=\phi-\phi_{0}$, so that:

$$
\frac{\partial \phi}{\partial t}=\frac{\partial \epsilon}{\partial t}=\lambda_{\mathrm{h}} \nabla^{2}(\delta p) ; \quad s \bar{\epsilon}=\lambda_{\mathrm{h}} \nabla^{2}(\delta \bar{p})
$$

The governing equations for the problem can be reduced to two equations in terms of the radial displacement $u$ (for an axisymmetric tunnel) and the pore pressure $p$. The first is obtained by observing that for a long plane strain tunnel, $\bar{\epsilon}=(\partial \bar{u} / \partial r)+(\bar{u} / r)$, therefore, following from Eq. (17) and since $\bar{u}, \delta \bar{p} \rightarrow 0$ as $r \rightarrow \infty$, we get:

$$
\bar{\epsilon}=\frac{\partial \bar{u}}{\partial r}+\frac{\bar{u}}{r}=\frac{\delta \bar{p}}{\omega(s)}
$$

Combining Eq. (20) with Eq. (17), we obtain the second governing equation:

$$
\nabla^{2}(\delta \bar{p})=q^{2} \delta \bar{p} ; \quad q=\sqrt{\frac{s}{\lambda_{\mathrm{h}} \omega(s)}}
$$

which expands for a plane strain axially symmetrical case to:

$$
\frac{\partial^{2}(\delta \bar{p})}{\partial r^{2}}+\frac{1}{r} \frac{\partial(\delta \bar{p})}{\partial r}=q^{2} \delta \bar{p}
$$

To complete the mathematical formulation of the tunnel problem, the initial and boundary conditions, to be discussed in the next section, must be specified.

\section{Initial and Boundary Conditions for Closing of the Tun- nel}

The life cycle of an underground tunnel may be represented by four stages as follows: (a) an initial phase of undisturbed in-situ ground which is in a state of hydromechanical equilibrium prior to the tunnel drilling (b) the tunnel is drilled into the ground medium and an internal support system is provided to maintain its stability during operations (c) at the end of its service life, the decommissioned tunnel is backfilled with a poroelastic material (d) the lining support degrades and eventually fails. For our study, the behaviour of the surrounding ground is assumed as poro-viscoelastic and the backfill as poroelastic, both fully saturated with fluid (water). The tunnel is supposed to be sufficiently deep (a depth of at least 10 times its diameter) so that the initial stress gradient can be neglected when analysing the near field behaviour. The tunnel is assumed to be perfectly circular to simplify analysis. Thus the problem consists of two zones, an inner zone which is the backfilled tunnel $(0 \leq r \leq a)$ and the outer zone which is the surrounding ground $(r \geq a)$. Though approximations are made, such a simplified model can provide useful orders of magnitude study of the mechanical behaviour of a real tunnel.

The initial state for the present study corresponds to the beginning to stage (d) of the tunnel life cycle, that is, the tunnel has been backfilled and closed off and the support starts to deteriorate. The backfill is assumed to be moderately compacted, so that the buildup of initial stress from compaction is supposed to be small. Since the effective stress from the self-weight of a few meters of backfill inside the tunnel will similarly be small in comparison to the overburden stresses in the host medium, the initial effective stress in the backfill may be assumed as null, but the initial pore pressure $p_{0}$ is in hydraulic equilibrium with the surrounding groundwater. Therefore, the initial conditions for the backfill are:

$$
\begin{aligned}
& 0 \leq r \leq a ; \quad u(r, 0)=0 ; \quad p(r, 0)=p_{0} \\
& \sigma_{\mathrm{ij}}(r, 0)=-p_{0} \delta_{\mathrm{ij}} ; \quad \varepsilon_{\mathrm{ij}}=0
\end{aligned}
$$

For the ground medium, it is assumed that the deviatoric stresses will have dissipated during stage (c) so that the stress field is isotropic. The initial conditions are therefore expressed as:

$$
\begin{aligned}
& a \leq r<\infty ; \quad u(r, 0)=0 ; \quad p(r, 0)=p_{0} ; \\
& \sigma_{\mathrm{ij}}(r, 0)=-\Sigma_{0} \delta_{\mathrm{ij}} ; \quad \varepsilon_{\mathrm{ij}}=0
\end{aligned}
$$

where $\Sigma_{0}$ stands for the initial overburden stress, assumed isotropic. This initial assumption, although not completely general, is made in order to simplify the theoretical developments and to make the problem amenable for the quasi-analytical solutions. It will be relaxed in subsequent developments, involving more complex material behaviors as well as more realistic geometries and workssequence. For these future developments, the quasi-analytical solutions presented here will serve as a very useful benchmark validation tool. The stress difference between the inner and outer zones at $r=a$ is initially taken up by the lining support:

$$
p_{\mathrm{s}}(t=0)=\Sigma_{0}-p_{0}
$$

The boundary conditions remain to be specified. There are two of them corresponding to the two independent variables $(u, p)$ of the governing equations. Substituting Eq. (19) into Eq. (18) and noting that $\epsilon=\phi-\phi_{0}$ gives,

$$
\frac{\partial \epsilon}{\partial t}=\operatorname{div}\left\{\lambda_{\mathrm{h}} \operatorname{grad}(\delta p)\right\}
$$

Since for infinitesimal strain, $\epsilon=\operatorname{div}(\mathbf{u})$, we obtain from Eq. (27),

$$
\operatorname{div}(\dot{\mathbf{u}})=\operatorname{div}\left\{\lambda_{\mathrm{h}} \operatorname{grad}(\delta p)\right\}
$$

Now $\operatorname{div}\{\operatorname{grad}(\delta p)\}=\nabla^{2}(\delta p)$ and for the case of a long plain strain circular tunnel, $\epsilon=\operatorname{div}(\mathbf{u})=\partial u / \partial r+u / r$ so that Eq. (28) reduces to:

$$
\frac{\partial \dot{u}}{\partial t}+\frac{\dot{u}}{r}=\frac{1}{r} \frac{\partial}{\partial r}(r \dot{u})=\lambda_{\mathrm{h}}\left\{\frac{1}{r} \frac{\partial}{\partial r}\left(r \frac{\partial(\delta p)}{\partial r}\right)\right\}
$$

where it is further assumed that the medium is homogeneous. After integrating Eq. (29) and noting that $u, \delta p$ vanishes when $r$ approaches $\infty$, we obtain the first boundary condition: 


$$
\left.\frac{\partial u}{\partial t}\right|_{\mathrm{r}=\mathrm{a}}=\left.\lambda_{\mathrm{h}} \frac{\partial(\delta p)}{\partial r}\right|_{\mathrm{r}=\mathrm{a}} ; \quad s \bar{u}(a, s)=\left.\lambda_{\mathrm{h}} \frac{\partial(\delta \bar{p})}{\partial r}\right|_{\mathrm{r}=\mathrm{a}}
$$

This boundary condition is a restatement of the requirement that for incompressible constituents, the convergence of the tunnel wall must be accompanied by the corresponding expulsion of an equivalent volume of water from the tunnel into the surrounding ground. The second boundary condition follows from the need to maintain equilibrium by means of the support $p_{\mathrm{s}}(t)$ provided by the lining:

$$
\sigma_{\mathrm{rr}}\left(a^{-}, t\right)-\sigma_{\mathrm{rr}}\left(a^{+}, t\right)=p_{\mathrm{s}}(t)
$$

Deterioration of the lining support will occur with elapsed time and this effect may be simulated by an exponential degradation law given by:

$$
p_{\mathrm{s}}(t)=\left(\Sigma_{0}-p_{0}\right) e^{-\kappa t}
$$

so that:

$$
\begin{aligned}
& \sigma_{\mathrm{rr}}\left(a^{-}, t\right)-\sigma_{\mathrm{rr}}\left(a^{+}, t\right)=\left(\Sigma_{0}-p_{0}\right) e^{-\kappa t} ; \\
& \bar{\sigma}_{\mathrm{rr}}\left(a^{-}, s\right)-\bar{\sigma}_{\mathrm{rr}}\left(a^{+}, s\right)=\frac{\left(\Sigma_{0}-p_{0}\right)}{s+\kappa}
\end{aligned}
$$

Details on the derivation of the interior radial stress of the lining support $\sigma_{\mathrm{rr}}\left(a^{-}, t\right)$ are presented in APPENDIX $\mathrm{A}$ and for the exterior radial stress $\sigma_{\mathrm{rr}}\left(a^{+}, t\right)$, in APPENDIX B.

\section{Analytical Solutions in Laplace Transform Space}

We present below the solutions of the ground $(r \geq a)$ for the governing Eqs. (21), (22) and the initial/boundary conditions Eqs. (24), (25), (30) and (31). The general solution of Eq. (22) for the pore pressure is known and can be written:

$$
\delta \bar{p}=A K_{0}(q r)+B I_{0}(q r)
$$

where $I_{0}, K_{0}$ are the modified Bessel functions of the first and second kind zero order respectively, and $A, B$ are constants. $B$ must vanish because $\delta \bar{p}$ tends to zero as $r \rightarrow$ $\infty$. Then, $A$ can be eliminated using Eq. (30) to give:

$$
\delta \bar{p}=-\bar{u}(a, s) \frac{s}{\lambda_{\mathrm{h}} q} \frac{K_{0}(q r)}{K_{1}(q a)}
$$

After manipulations detailed in APPENDIX A and B, Eq. (33) can be used to obtain:

$$
\bar{u}(a, s)=-\frac{\kappa a}{s(s+\kappa)} \frac{\Sigma_{0}-p_{0}}{\Omega(s)}
$$

where

$$
\Omega(s)=\left(2 K_{\mathrm{R}}+\frac{2}{3} \mu_{\mathrm{R}}+2 s \bar{\mu}\right)+\frac{a s}{\lambda_{\mathrm{h}} q} \frac{K_{0}(q a)}{K_{1}(q a)}
$$

Finally:

$$
\delta \bar{p}=\frac{\kappa a\left(\Sigma_{0}-p_{0}\right)}{(s+\kappa) \lambda_{\mathrm{h}} q \Omega(s)} \frac{K_{0}(q r)}{K_{1}(q a)}
$$

To obtain the displacement field, we substitute Eq. (35) into Eq. (21) and integrate for $\bar{u}$ while ensuring that the constants of integration must satisfy the displacement at $r$ $=a$, hence:

$$
\bar{u}(r, s)=-\frac{\kappa a\left(\Sigma_{0}-p_{0}\right)}{s(s+\kappa) \Omega(s)} \frac{K_{1}(q r)}{K_{1}(q a)}
$$

The radial stress, $\sigma_{\mathrm{rr}}=\sigma+s_{\mathrm{rr}}$, is the additive sum of the mean stress and the deviatoric stress. Noting that $\sigma^{0}=$ $-\Sigma_{0}, s_{\mathrm{ij}}^{0}=0$, then from Eqs. (3) and (4) we get:

$$
\bar{\sigma}=-\frac{\Sigma_{0}}{s}+s \bar{K} \bar{\epsilon}-\delta \bar{p} ; \quad \bar{S}_{\mathrm{rr}}=2 s \overline{\mu e}_{\mathrm{rr}}
$$

Thus, keeping in mind that $\bar{e}_{\mathrm{rr}}=\bar{\varepsilon}_{\mathrm{rr}}-\bar{\epsilon} / 3, \bar{\varepsilon}_{\mathrm{rr}}=\bar{\epsilon}-\bar{u} / r$, after some manipulation it is found that:

$$
\bar{\sigma}_{\mathrm{rr}}(r, s)=-\frac{\Sigma_{0}}{s}-2 s \bar{\mu} \frac{\bar{u}}{r}
$$

The circumferential (hoop) stress $\sigma_{\theta \theta}$ and the axial stress $\sigma_{\mathrm{zz}}$ can be derived in the same manner giving:

$$
\begin{aligned}
& \bar{\sigma}_{\theta \theta}(r, s)=-\frac{\Sigma_{0}}{s}+2 s \bar{\mu}\left(\frac{\bar{u}}{r}-\frac{\delta \bar{p}}{\omega(s)}\right) ; \\
& \bar{\sigma}_{\mathrm{zz}}(r, s)=-\frac{\Sigma_{0}}{s}-2 s \bar{\mu} \frac{\delta \bar{p}}{\omega(s)}
\end{aligned}
$$

Note that the solutions (Eqs. (35)-(41)) do not limit the choices for $K, \mu$, but for the particular case of Eqs. (7) and (8), it is observed that when the viscosity becomes very large (which can be symbolically expressed as viscosity $\rightarrow \infty$ ) so that the characteristic times $\tau_{\mathrm{r}}, \theta_{\mathrm{r}}, \tau_{\mathrm{c}}, \theta_{\mathrm{c}}$ tend to infinity, the ground converges toward a poroelastic behaviour and:

$$
\begin{aligned}
& \omega_{\mathrm{e}}=\lim _{\text {viscosity } \rightarrow \infty} \omega=K_{0}+\frac{4}{3} \mu_{0} ; \\
& \Omega_{\mathrm{e}}=\lim _{\text {viscosity } \rightarrow \infty} \Omega=2 K_{\mathrm{R}}+\frac{2}{3} \mu_{\mathrm{R}}+2 \mu_{0} ; \\
& \lim _{\text {viscosity } \rightarrow \infty} q(s)=\sqrt{\frac{s}{\lambda_{\mathrm{h}} \omega_{\mathrm{e}}}}
\end{aligned}
$$

\section{Asymptotic Solutions at Large Times $(t \rightarrow \infty)$}

To derive the asymptotic solutions at large times as $t \rightarrow$ $\infty$, we invoke the classic Laplace transform theorem

$$
\lim _{s \rightarrow 0} s \bar{f}(s)=\lim _{t \rightarrow \infty} f(t) .
$$

The following can also be established:

$$
\begin{aligned}
& \omega(0)=K_{\infty}+\frac{4}{3} \mu_{\infty} ; \Omega(0)=2 K_{\mathrm{R}}+\frac{2}{3} \mu_{\mathrm{R}}+2 \mu_{\infty} ; \lim _{s \rightarrow 0} q(s)=0 \\
& \lim _{s \rightarrow 0} q K_{1}(q r)=\frac{1}{r} ; \quad \lim _{s \rightarrow 0} s K_{0}(q r)=0 ; \quad \lim _{s \rightarrow 0} s \bar{\mu}=\mu_{\infty}
\end{aligned}
$$

Combining Eq. (43) with Eqs. (35) and (38) it is evident that:

$$
\delta p(r, \infty)=0 ; \quad u(r, \infty)=-\frac{a^{2}}{r} \frac{\Sigma_{0}-p_{0}}{\left(2 K_{\mathrm{R}}+\frac{2}{3} \mu_{\mathrm{R}}+2 \mu_{\infty}\right)}
$$

Hence, eventually $p(r, \infty)=p_{0}$, hydraulic equilibrium is 
restored at the initial pore pressure while the radial displacement varies inversely as the radial distance. Use of Eqs. (40), (41), (43) and (44) further results in the following asymptotic total stresses at large times:

$$
\begin{aligned}
& \sigma_{\mathrm{rr}}(r, \infty)=-\Sigma_{0}-2 \mu_{\infty} \frac{u(r, \infty)}{r} ; \\
& \sigma_{\theta \theta}(r, \infty)=-\Sigma_{0}+2 \mu_{\infty} \frac{u(r, \infty)}{r} ; \quad \sigma_{\mathrm{zz}}(r, \infty)=-\Sigma_{0} \\
& \sigma_{\mathrm{rr}}(r, \infty)-\sigma_{\theta \theta}(r, \infty)=-4 \mu_{\infty} \frac{u(r, \infty)}{r}
\end{aligned}
$$

It is also noted that the deviatoric stress varies inversely as the square of the radial distance.

\section{Solutions in Dimensionless Form}

The solutions obtained above will be normalized to a dimensionless form. The radius of the tunnel, $a$, is chosen as the characteristic length, the initial overburden stress $\Sigma_{0}$ is the characteristic stress or pressure and the characteristic diffusion time is taken as (Wong et al., 2008a),

$$
\tau_{\mathrm{h}}=\frac{a^{2}}{\lambda_{\mathrm{h}} \omega_{\mathrm{e}}} ; \quad \omega_{\mathrm{e}}=K_{0}+\frac{4}{3} \mu_{0}
$$

Using the above characteristic quantities, we now define the following dimensionless parameters (with an asterisk superscript) in Table 1.

The definitions shown in Table 1 lead to the following

expressions:

$$
\begin{aligned}
& \omega^{*}\left(s^{*}\right)=s^{*}\left(\bar{K}^{*}+\frac{4}{3} \bar{\mu}^{*}\right) ; \\
& \Omega^{*}\left(s^{*}\right)=2 K_{\mathrm{R}}^{*}+\frac{2}{3} \mu_{\mathrm{R}}^{*}+2 s^{*} \bar{\mu}^{*}+\frac{\omega_{\mathrm{S}}^{*} s^{*}}{q^{*}} \frac{K_{0}\left(q^{*}\right)}{K_{1}\left(q^{*}\right)} \\
& \bar{K}^{*}\left(s^{*}\right)=\frac{K_{0}^{*}}{s^{*}}\left\{\frac{K_{\infty}^{*}}{K_{0}^{*}}+\frac{s^{*}\left(1-K_{\infty}^{*} / K_{0}^{*}\right)}{\left(s^{*}+1 / \tau_{\mathrm{r}}^{*}\right)}\right\} ; \\
& \bar{\mu}^{*}\left(s^{*}\right)=\frac{\mu_{0}^{*}}{s^{*}}\left\{\frac{\mu_{\infty}^{*}}{\mu_{0}^{*}}+\frac{s^{*}\left(1-\mu_{\infty}^{*} / \mu_{0}^{*}\right)}{\left(s^{*}+1 / \theta_{\mathrm{r}}^{*}\right)}\right\}
\end{aligned}
$$

and the solutions expressed in the dimensionless form

\begin{tabular}{|c|c|c|c|}
\hline $\begin{array}{l}\text { Dimensionless } \\
\text { parameters }\end{array}$ & Definition/Comments & $\begin{array}{l}\text { Dimensionless } \\
\text { parameters }\end{array}$ & Definition/Comments \\
\hline$r^{*}=\frac{r}{a}$ & Radius & $\mu_{\mathrm{R}}^{*}=\frac{\mu_{\mathrm{R}}}{\Sigma_{0}}$ & Shear modulus of backfill \\
\hline$u^{*}=\frac{u}{a}$ & Radial displacement & $s^{*}=s \tau_{\mathrm{h}}$ & Laplace transform parameter \\
\hline$t^{*}=\frac{t}{\tau_{\mathrm{h}}}$ & Time & $\omega^{*}\left(s^{*}\right)=\frac{\omega(s)}{\Sigma_{0}}$ & $\begin{array}{l}\text { A parameter related to the bulk and shear } \\
\text { relaxation kernels }\end{array}$ \\
\hline$\kappa^{*}=\kappa \tau_{\mathrm{h}}$ & Liner degradation rate & $\omega_{\mathrm{e}}^{*}=\frac{\omega_{\mathrm{e}}}{\Sigma_{0}}$ & Limiting value of $\omega^{*}$ as viscosity $\rightarrow \infty$ \\
\hline$p^{*}=\frac{p}{\Sigma_{0}}$ & Pore pressure & $\Omega^{*}\left(s^{*}\right)=\frac{\Omega(s)}{\Sigma_{0}}$ & $\begin{array}{l}\text { A parameter related to backfill and ground } \\
\text { properties }\end{array}$ \\
\hline$K^{*}=\frac{K}{\Sigma_{0}}$ & Bulk relaxation kernel & $\Omega_{\mathrm{e}}^{*}=\frac{\Omega_{\mathrm{e}}}{\Sigma_{0}}$ & Limiting value of $\Omega^{*}$ as viscosity $\rightarrow \infty$ \\
\hline$K_{\infty}^{*}=\frac{K_{\infty}}{\Sigma_{0}}$ & Long term bulk modulus of ground & $\tau_{\mathrm{c}}^{*}=\frac{\tau_{\mathrm{c}}}{\tau_{\mathrm{h}}}$ & Bulk characteristic creep time \\
\hline$K_{0}^{*}=\frac{K_{0}}{\Sigma_{0}}$ & $\begin{array}{l}\text { Short term (instantaneous) bulk modulus of } \\
\text { ground }\end{array}$ & $\tau_{\mathrm{r}}^{*}=\frac{\tau_{\mathrm{r}}}{\tau_{\mathrm{h}}}$ & Bulk characteristic relaxation time \\
\hline$\mu^{*}=\frac{\mu}{\Sigma_{0}}$ & Shear relaxation kernel & $\theta_{\mathrm{c}}^{*}=\frac{\theta_{\mathrm{c}}}{\tau_{\mathrm{h}}}$ & Shear characteristic creep time \\
\hline$\mu_{\infty}^{*}=\frac{\mu_{\infty}}{\Sigma_{0}}$ & Long term shear modulus of ground & $\theta_{\mathrm{r}}^{*}=\frac{\theta_{\mathrm{r}}}{\tau_{\mathrm{h}}}$ & Shear characteristic relaxation time \\
\hline$\mu_{0}^{*}=\frac{\mu_{0}}{\Sigma_{0}}$ & $\begin{array}{l}\text { Short term (instantaneous) shear modulus of } \\
\text { ground }\end{array}$ & $q^{*}=\sqrt{\frac{\omega_{\mathrm{e}}^{*} s^{*}}{\omega^{*}\left(s^{*}\right)}}=q a$ & A parameter dependent on $\omega_{\mathrm{e}}^{*}, \omega^{*}\left(s^{*}\right)$ and $s^{*}$ \\
\hline$K_{\mathrm{R}}^{*}=\frac{K_{\mathrm{R}}}{\Sigma_{0}}$ & Bulk modulus of backfill & & \\
\hline
\end{tabular}
are:

$$
\begin{aligned}
& \delta \bar{p}^{*}=\frac{\kappa^{*} \omega_{\mathrm{e}}^{*}\left(1-p_{0}^{*}\right)}{\left(s^{*}+\kappa^{*}\right) q^{*} \Omega^{*}\left(s^{*}\right)} \frac{K_{0}\left(q^{*} r^{*}\right)}{K_{1}\left(q^{*}\right)} \\
& \bar{u}^{*}=-\frac{\kappa^{*}\left(1-p_{0}^{*}\right)}{s^{*}\left(s^{*}+\kappa^{*}\right) \Omega^{*}\left(s^{*}\right)} \frac{K_{1}\left(q^{*} r^{*}\right)}{K_{1}\left(q^{*}\right)} \\
& \bar{\sigma}_{\mathrm{rr}}^{*}=-\frac{1}{s^{*}}-2 s^{*} \bar{\mu}^{*} \frac{\bar{u}^{*}}{r^{*}} \\
& \bar{\sigma}_{\theta \theta}^{*}=-\frac{1}{s^{*}}+2 s^{*} \bar{\mu}^{*}\left(\frac{\bar{u}^{*}}{r^{*}}-\frac{\delta \bar{p}^{*}}{\omega^{*}\left(s^{*}\right)}\right) ; \\
& \bar{\sigma}_{z z}^{*}=-\frac{1}{s^{*}}-2 s^{*} \bar{\mu}^{*} \frac{\delta \bar{p}^{*}}{\omega^{*}\left(s^{*}\right)}
\end{aligned}
$$

Table 1. Definition of dimensionless parameters 


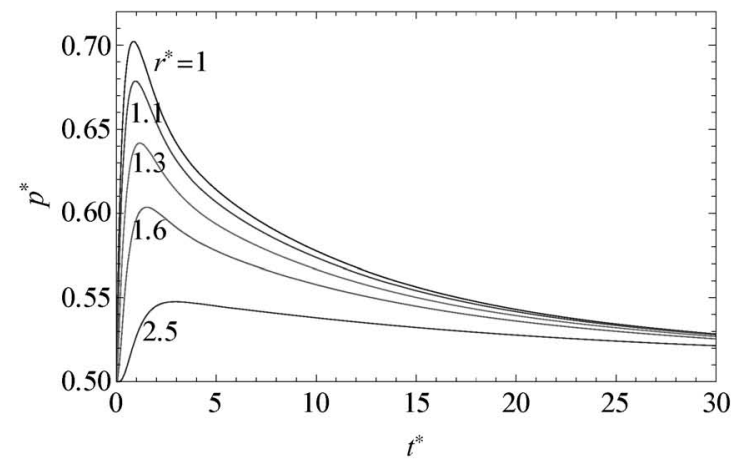

(a)

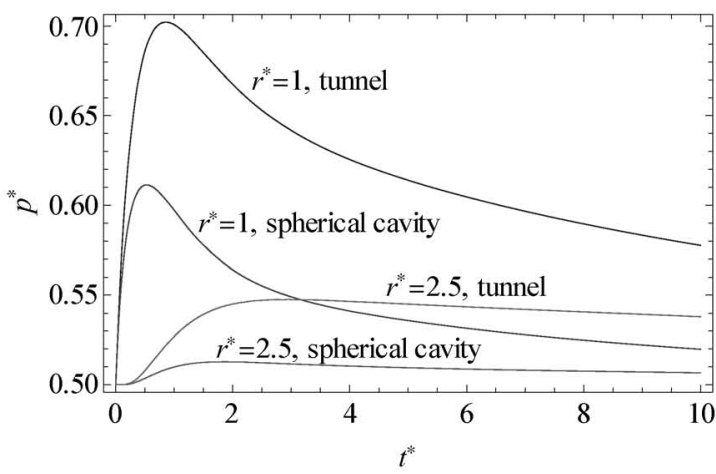

(b)

Fig. 3. (a) Evolution of normalized pore pressure in the ground medium of the tunnel with time at $r^{*}=1,1.1,1.3,1.6$ and 2.5 and $(b)$ comparison of the normalized pressure for tunnel and spherical cavity at $r^{*}=1$ and 2.5

\section{NUMERICAL STUDY}

For the purpose of the present study we have chosen the same set of reference parameter values adopted in our earlier paper (Wong et al., 2008a) and inspired by Gaombalet (2004):

$$
\begin{aligned}
& \Sigma_{0}=10 \mathrm{MPa} ; \quad p_{0}=5 \mathrm{MPa} ; \quad K_{0}=1680 \mathrm{MPa} ; \\
& \mu_{0}=1890 \mathrm{MPa} ; \quad K_{\mathrm{R}}=K_{0} / 30 ; \\
& \lambda_{\mathrm{h}}=2.4 \times 10^{-17} \mathrm{~m}^{4} \mathrm{~N}^{-1} \mathrm{~s}^{-1} ; \quad K_{\infty}=560 \mathrm{MPa} ; \\
& \mu_{\infty}=630 \mathrm{MPa} ; \quad \kappa^{*}=2 ; \quad \tau_{\mathrm{r}}^{*}=4 ; \quad \theta_{\mathrm{r}}^{*}=2
\end{aligned}
$$

In addition we chose $\mu_{\mathrm{R}}=\mu_{0} / 30$, keeping to same proportion for the shear modulus as the bulk modulus. This is to represent the physical state where the backfill stiffness is less than those of the surrounding ground. From the above we get:

$$
\begin{array}{lll}
p_{0}^{*}=0.5 ; & K_{0}^{*}=168 ; & \mu_{0}^{*}=189 ; \quad K_{\mathrm{R}}^{*}=5.6 ; \\
\mu_{\mathrm{R}}^{*}=6.3 ; & K_{\infty}^{*}=56 ; & \mu_{\infty}^{*}=63
\end{array}
$$

The solutions obtained in the time domain have been numerically inverted using the Stefhest algorithm (1970) as none are analytically invertible except for the asymptotic cases. The Stefhest algorithm is one of the numerous numerical schemes available to evaluate the integration in Eq. (2) numerically. Figure 3(a) shows the evolution of the normalised pore pressure with time at $r^{*}=1,1.1,1.3$, 1.6 and 2.5. The pore pressure peak increases and the time to peak are shorter as the radial distance edges closer to the tunnel wall. As will be seen later in Fig. 6(a), the peak value depends significantly on the rate of lining deterioration. After reaching the peak, the pressure dissipates gradually with time until eventually reaching equilibrium at the initial pore pressure. A comparison of the pressure evolution for the cases of a tunnel and of a spherical cavity is provided in Fig. 3(b). The magnitude of the pressure peak is smaller, the time to peak is shorter and the dissipation of excess pore pressure is faster for the spherical cavity. These results suggest that the characteristic response time for the spherical cavity is shorter, namely, the hydromechanical interactions occur more rapidly in the spherical cavity case relative to a long tunnel. One could be tempted to think that the explanation lays entirely in the difference of the ratio between the volume of the opening and its surface area of exchange with the ground, but the following figures, notably Fig. 4(c), illustrate a more complex reality. Indeed, it would have been difficult to anticipate the smaller pressure peak found in the spherical cavity without undertaking the appropriate analysis.

In Fig. 4(a), the evolution of normalized radial displacement $u^{*}\left(r^{*}, t\right) / u^{*}(1, \infty)$ with time is shown. The normalization in terms of the long term radial convergence at the wall/ground interface $\left(r^{*}=1\right)$ confines the value to the interval $(0,1)$. Shown on the same plot, in Fig. 4(b), are the radial convergences for the tunnel and the spherical cavity at $r^{*}=1$ and 2.5. The displacements for the spherical cavity are also normalised with respect to the long term radial convergence at the cavity wall/ground interface, which confines both the values for the tunnel and the spherical cavity to the interval $(0,1)$ despite a difference between actual displacements. It can be surmised that relative to the tunnel, the spherical cavity converges more rapidly to the long term values. Figure 4(c) shows the plot of the long term radial displacements, $u^{*}\left(r^{*}, \infty\right)$, against the radial distance for different tunnel backfill shear moduli $\mu_{\mathrm{R}}^{*}=6.3,56$ and 560 . The results for this case are intuitively predictable since, as the shear modulus of the backfill is increased, there is a corresponding reduction in the radial convergence, whereas it may be recalled that for a spherical cavity the backfill shear modulus has no effect on the responses. It can be deduced from Eq. (44) that $u^{*}\left(r^{*}, \infty\right)$ varies inversely as the radial distance for the tunnel, but as the square of the radial distance for the spherical cavity, so that for the latter, the long term convergence values diminishes more quickly with $r^{*}$. As a consequence, the convergence displacements for a spherical cavity generally appear more confined in the area of the sidewall. The arching effect is evidently greater for the spherical cavity than it is for a similar tunnel.

The evolution of the normalised effective radial stress 


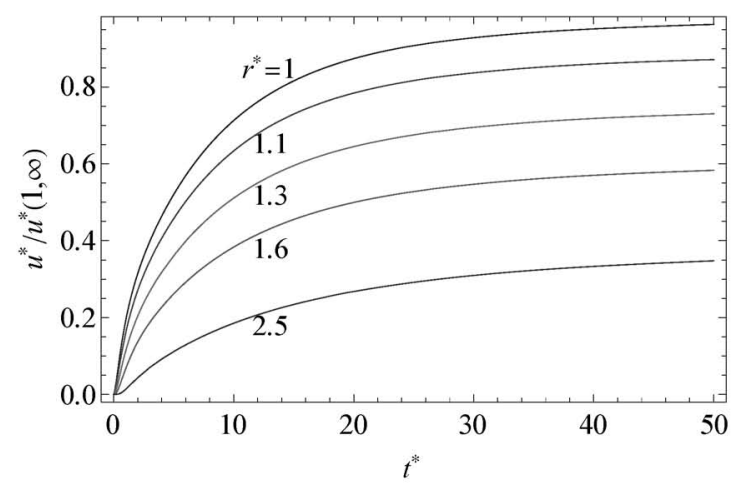

(a)

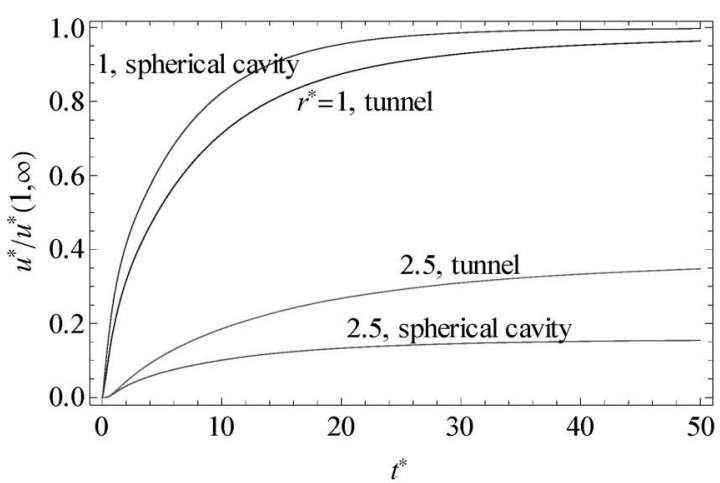

(b)

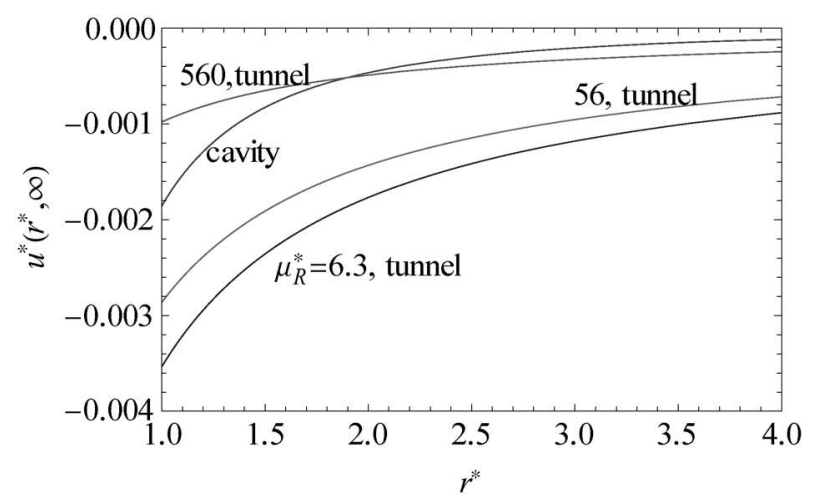

(c)

Fig. 4. (a) Evolution of normalized radial convergence $\left(u^{*}\left(r^{*}, t\right) / u^{*}(1, \infty)\right)$ of the tunnel with time at $r^{*}=1,1.1,1.3,1.6$ and 2.5 , (b) comparison of the normalized radial convergence for tunnel and spherical cavity at $r^{*}=1$ and 2.5 and (c) the long term tunnel convergence for the radial interval $r^{*}=(1,4)$ and $\mu_{\mathrm{R}}^{*}=6.3,56$ and 560 . The long term spherical cavity convergence is also shown

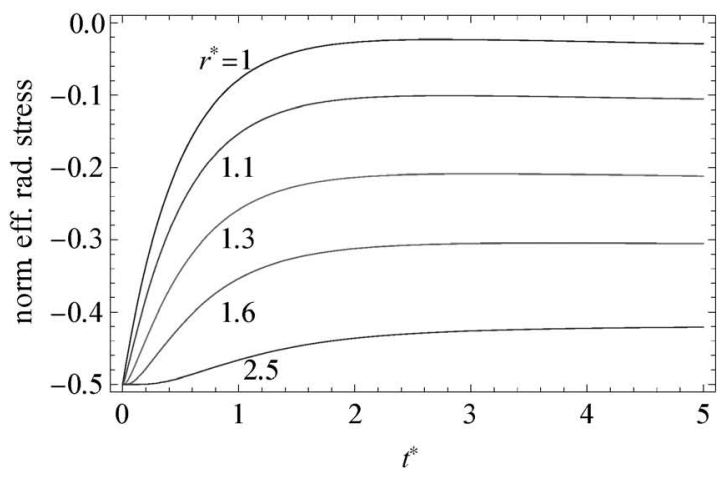

(a)

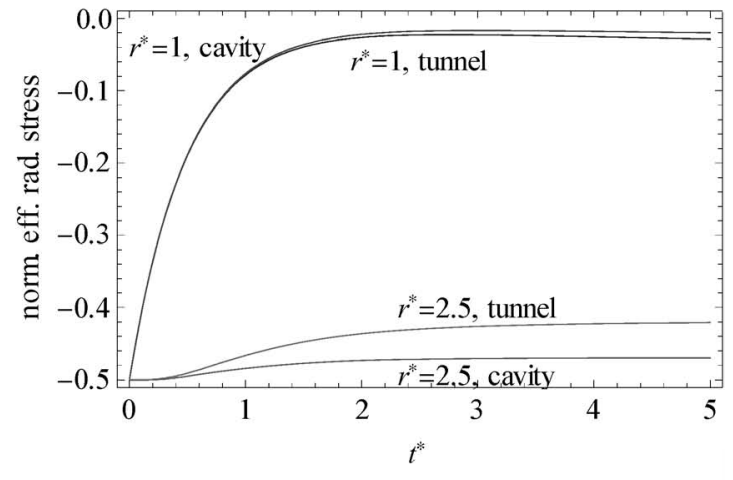

(b)

Fig. 5. (a) Evolution of normalized effective radial stress in the ground medium of the tunnel with time at $r^{*}=1,1.1,1.3,1.6$ and 2.5 and (b) comparison of the normalized effective radial stress convergence for tunnel and spherical cavity at $r^{*}=1$ and 2.5

with time for the tunnel shown in Fig. 5(a) reflects the trend occurring in Fig. 4(a) for tunnel convergence but on a shorter time scale. A comparison between tunnel and spherical cavity for the effective radial stress, Fig. 5(b), also reflects the observations made for Fig. 4(b). These results can be anticipated since the radial displacement is a function of the effective radial stress.
Figure 6(a) shows the effects of the lining deterioration rate $\kappa^{*}$ of the tunnel where virtually instantaneous lining failure can be simulated by setting $\kappa^{*}$ to a very large value (in this case a value of 500). Hence, it is observed that an immediate surge in pore pressure at the tunnel wall $\left(r^{*}=\right.$ 1) is obtained for $\kappa^{*}=500$ as the additional stress from the surrounding ground resulting from the lining failure 


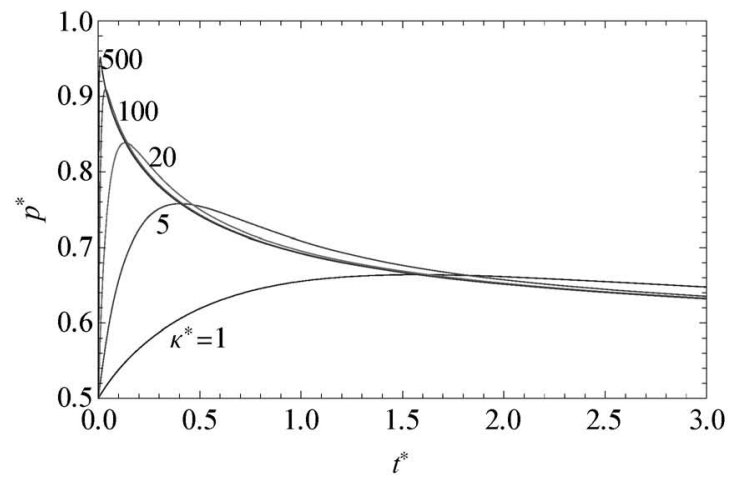

(a)

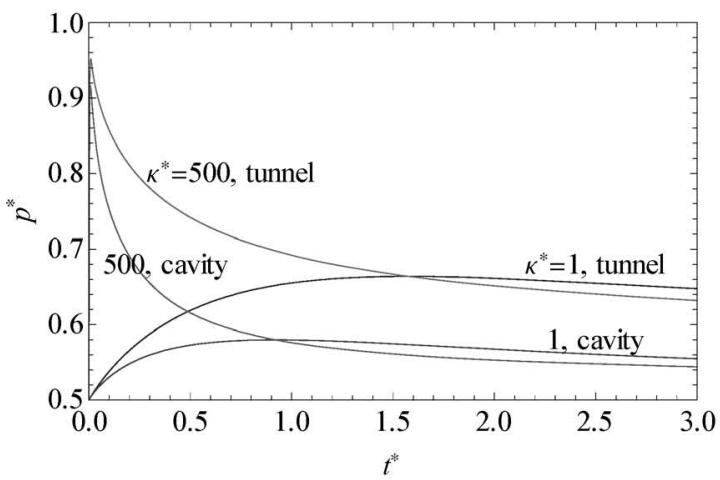

(b)

Fig. 6. (a) Evolution of normalized pore pressure at the tunnel wall $\left(r^{*}=1\right)$ with time for lining deterioration rate $\kappa^{*}=1,5,20,100$ and 500 and $(b)$ comparison of the normalized pore pressure at walls of tunnel and spherical cavity $\left(r^{*}=1\right)$ for contrasting lining deterioration rate $\kappa^{*}=1$ and 500

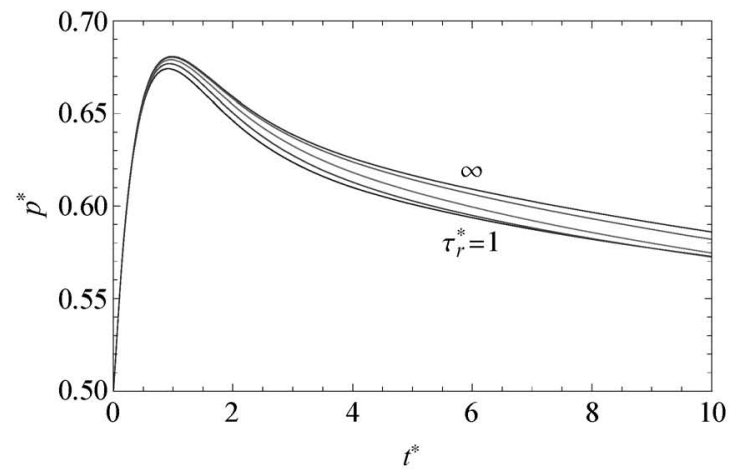

(a)

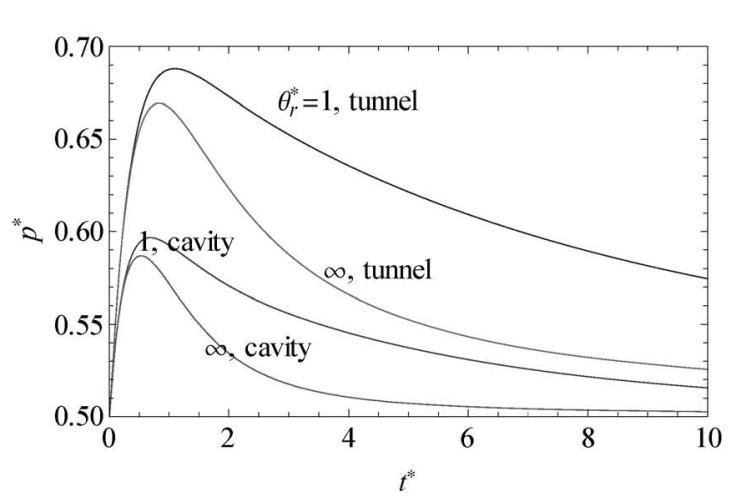

(c)

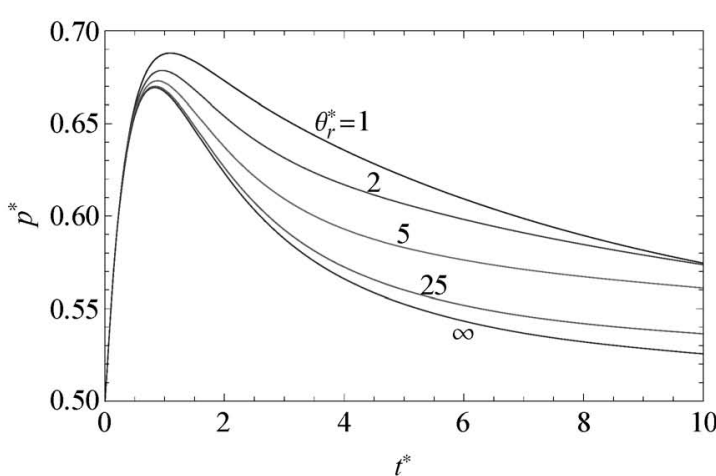

(b)

Fig. 7. (a) Evolution of normalized pore pressure in the ground medium of the tunnel with time at $r^{*}=1.1$ and increasing characteristic relaxation times $\tau_{\mathrm{r}}^{*}=1,2,5,25, \infty$, (b) evolution of normalized pore pressure in the ground medium of the tunnel with time at $r^{*}=1.1$ for characteristic relaxation times $\theta_{\mathrm{r}}^{*}=1,2,5,25, \infty$ and (c) normalized pore pressure for tunnel and spherical cavity at $r^{*}=1.1$ and contrasting characteristic relaxation times $\theta_{\mathrm{r}}^{*}=1$ and $\infty$

is immediately taken up by the pore water. Conversely; a slow lining deterioration rate, $\kappa^{*}=1$, shows a smothered peak. It is evident from these results that $\kappa^{*}$ has a large effect on the pore pressure peak, namely, the faster the rate of lining deterioration, the higher and sooner a pressure peak can occur. It may also be observed that $p^{*}$ eventually asymptotes to the equilibrium state $\left(p^{*}=0.5\right)$ as $t^{*} \rightarrow \infty$ in each case. Figure 6(b) further confirms that the responses for a spherical cavity are more rapid than for a tunnel.

The viscous phenomenon from creep are shown in Figs. 7(a), (b) and (c) where the plots are obtained for the 


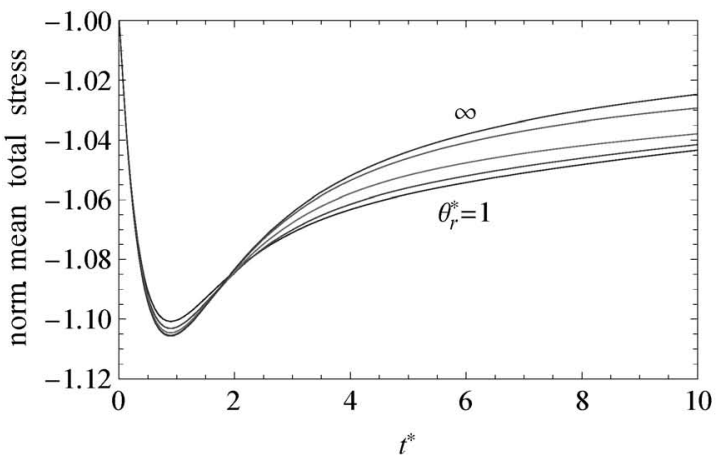

(a)

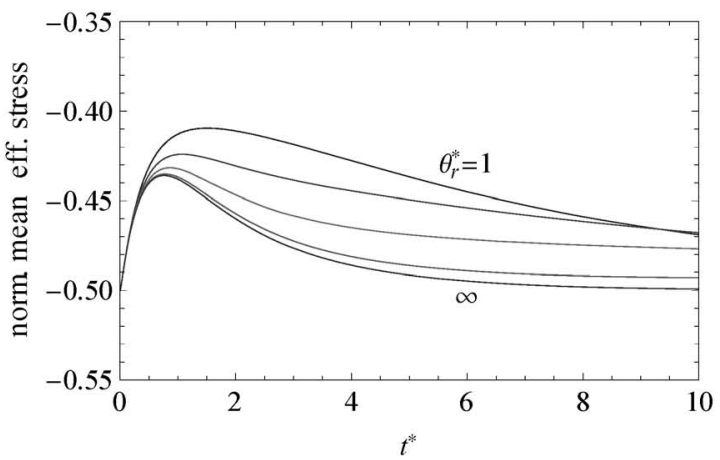

(b)

Fig. 8. (a) Evolution of normalized mean total stress in the ground medium of the tunnel at $\boldsymbol{r}^{*}=1.1$ with time for characteristic relaxation times $\theta_{\mathrm{r}}^{*}$ $=1,2,5,25, \infty$ and (b) normalized mean effective stress of tunnel for characteristic relaxation times $\theta_{\mathrm{r}}^{*}=1,2,5,25, \infty$

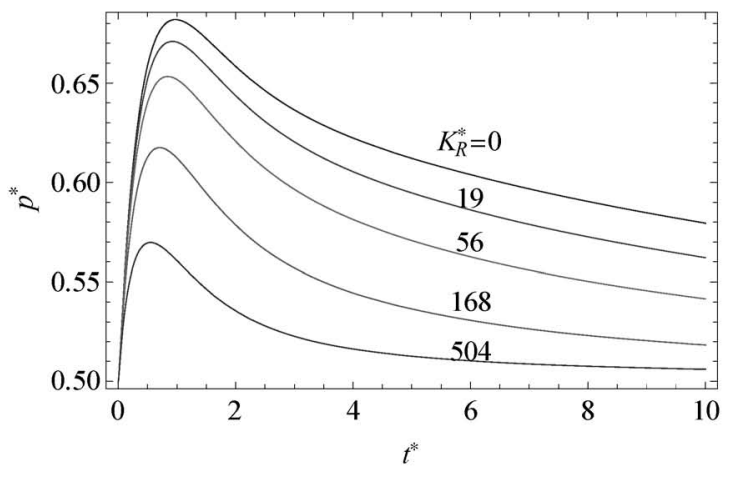

(a)

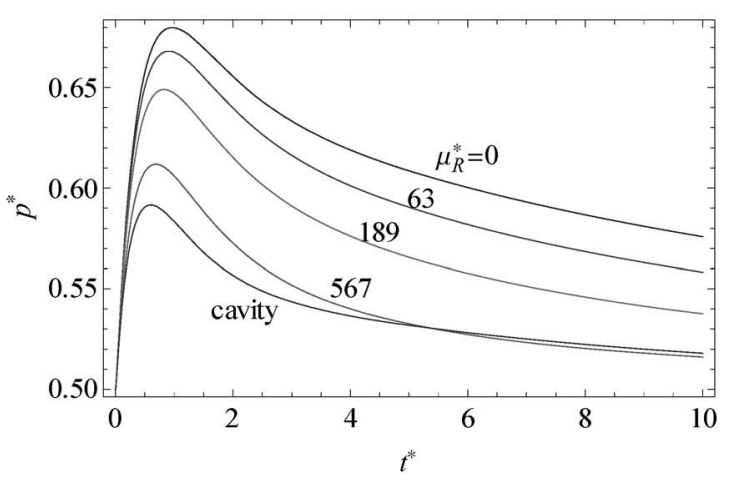

(b)

Fig. 9. (a) Evolution of normalized pore pressure in the ground medium of the tunnel at $r^{*}=1.1$ with time for backfill bulk modulus $K_{\mathrm{R}}^{*}=0$, 19, 56, $168,504,(b)$ for tunnel backfill shear modulus, $\mu_{\mathrm{R}}^{*}=0,21,63,189,567$, and spherical cavity case and (c) normalized pore pressure for tunnel and spherical cavity at contrasting bulk modulus $K_{\mathrm{R}}^{*}=0$ and $504 ; \mu_{\mathrm{R}}^{*}=0$ and 567

normalized pore pressure at $r^{*}=1.1$ representing a point in the near field of the tunnel. It is at once observed in Fig. 7(a) that the characteristic relaxation time of the bulk modulus, $\tau_{\mathrm{r}}^{*}$, only has a marginal effect on the pore pressure and by extension, on the convergence and stress responses. A similar finding is reported in Wong et al. (2008a) regarding this effect for the spherical cavity. Conversely, the characteristic relaxation time of the shear modulus, $\theta_{\mathrm{r}}^{*}$, has a more significant effect on the pore pressure (Fig. 7(b)). As $\theta_{\mathrm{r}}^{*}$ tends to infinity (while keeping $\mu_{0}^{*}=56, \mu_{\infty}^{*}=63$ ), creep vanishes to zero and the response converges to the elastic solution whereby $\mu^{*} \rightarrow \mu_{0}^{*}$. Thus, creep has the effect of both delaying and increasing the transient pressure peak while slowing its dissipation to equilibrium in time. In Fig. 7(c) a comparison is made between the tunnel and spherical cavity showing the comparatively more acute response for a tunnel (higher pressure peak, slower dissipation) than for a spherical cavity.

The transient mean total stress of the tunnel at $r^{*}=1.1$ and for a range of characteristic relaxation times $\theta_{\mathrm{r}}^{*}$ are shown in Fig. 8(a). The minima of the mean total stress is only marginally influenced by the effect of viscosity, and the stress dissipation (ultimately converging to a value of
1) is slower at a smaller relaxation time $\theta_{\mathrm{r}}^{*}$. By contrast, in Fig. 8(b), it is shown that $\theta_{\mathrm{r}}^{*}$ has a greater effect on the transient mean effective stress peak as well as the stress dissipation, namely, a smaller $\theta_{\mathrm{r}}^{*}$ will give rise to a higher peak and slower dissipation (eventually to a value of 0.5 ).

The effects on the pore pressure with respect to time due to the changes in the bulk $\left(K_{\mathrm{R}}^{*}\right)$ and shear $\left(\mu_{\mathrm{R}}^{*}\right)$ moduli of the backfill, are presented in Figs. 9(a) and (b). $K_{\mathrm{R}}^{*}=0$ and/or $\mu_{\mathrm{R}}^{*}=0$ corresponds to the case where there is no backfill, i.e. a non backfilled tunnel only full of water. By contrast, $K_{\mathrm{R}}^{*}=504$ corresponds to a backfill which is 3 times stiffer than short term bulk modulus $K_{0}^{*}$, while $\mu_{\mathrm{R}}^{*}=$ 567 is 3 times the short term shear modulus $\mu_{0}^{*}$. It is observed that the more the stiffness of the backfill (in both bulk and/or shear modulus) increases, the more it will be able to take up any stress increase. Consequently, the transient pore pressure peak will be smaller and the excess pore pressure will dissipate more rapidly. Further, it is noted that the shear modulus of the backfill has no effect on the pore pressure responses for a spherical cavity. In Fig. 9(b), where the responses for the tunnel with backfill shear modulus $\mu_{\mathrm{R}}^{*}=0,63,189,567$ are plotted, it is evident that $\mu_{\mathrm{R}}^{*}$ has a significant effect on the pore pressure 


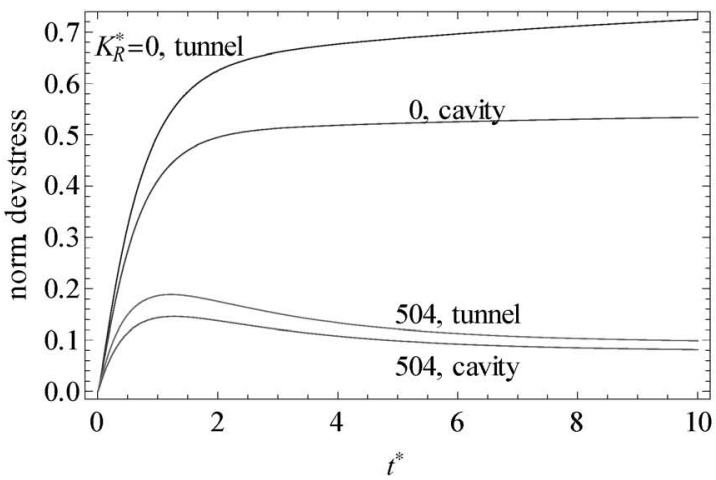

(a)

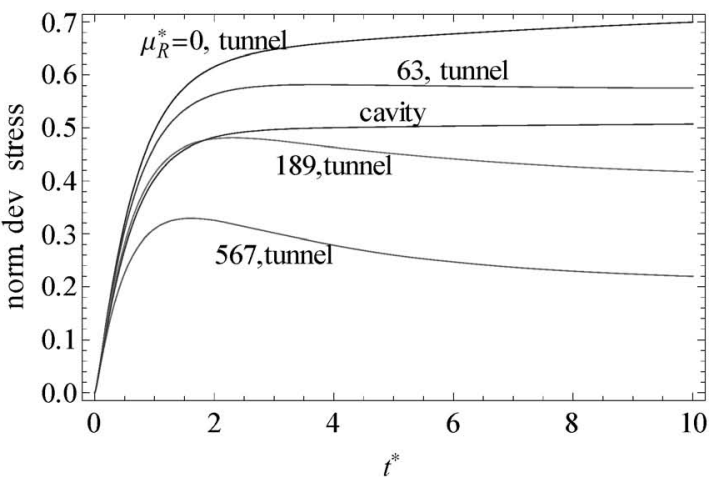

(b)

Fig. 10. Comparison of evolution of normalized deviatoric stress in the ground medium of the tunnel and spherical cavity at $r^{*}=1.1$ with time for: (a) backfill bulk modulus $K_{\mathrm{R}}^{*}=0$ and (b) tunnel backfill shear modulus $\mu_{\mathrm{R}}^{*}=0,63,189,567$, and spherical cavity (which is not affected by backfill shear modulus)

for a tunnel, although it has none for a spherical cavity. A comparison between the pore pressure responses between tunnel and spherical cavity at contrasting backfill bulk modulus, $K_{\mathrm{R}}^{*}=0$ and 504 , can be drawn from the plots in Fig. 9(b). This confirms earlier results that the quality of the backfill can have an even more important effect in attenuating the transient pore pressure peak and in increasing the rate of pore pressure dissipation for a long tunnel than for a spherical cavity.

Shown in Figs. 10(a) and (b) is a comparison of the evolution of the normalized deviatoric stress with time at the radial distance $r^{*}=1.1$ between the tunnel and the spherical cavity. The backfill bulk modulus is $K_{\mathrm{R}}^{*}=0$ and 504 for both tunnel and cavity, and the shear modulus, $\mu_{\mathrm{R}}^{*}=0,63,189,567$ for the tunnel. $K_{\mathrm{R}}^{*}$ has a significant effect on the deviatoric stresses for both the tunnel and spherical cavity as well as $\mu_{\mathrm{R}}^{*}$ for the tunnel. Depending on the values of $K_{\mathrm{R}}^{*}$ and/or $\mu_{\mathrm{R}}^{*}$, the deviatoric stresses converge to the equilibrium values following a transient period of interaction between the backfill and the surrounding ground. It is observed that for the stiffer backfill, the equilibrium deviatoric stresses are lower for both the tunnel and the spherical cavity so that dissipation to the final equilibrium values takes a longer time to occur.

\section{CONCLUSIONS}

Quasi-analytical solutions have been developed to study the hydromechanical responses in terms of pore pressure, stress and displacements caused by the post closure behaviour of a long underground circular tunnel where the surrounding ground is a saturated poro-viscoelastic medium and the backfill a saturated poroelastic material. The solutions developed are analytic in the Laplace transform space and can be efficiently inverted numerically by means of an inversion algorithm (e.g., Stehfest, 1970; Talbot, 1979). The responses have been studied particularly in relation to the backfill stiffness and the deterioration rate of the lining. Globally, the results obtained are almost qualitatively similar to those for a spherical cavity found in earlier paper (Wong et al., 2008a). Nevertheless, it can be pointed out that for a tunnel, the characteristic time of the hydromechanical responses will be longer while the perturbed zone will be more spread out than for a spherical cavity of identical radius. The backfill shear modulus has no influence on the spherical cavity responses whereas it has a significant effect for a tunnel. This study shows that, generally speaking, the backfill stiffness $\left(K_{\mathrm{R}}^{*}\right.$ and $\left.\mu_{\mathrm{R}}^{*}\right)$ and the deterioration rate of the lining support $\left(\kappa^{*}\right)$ have more significant implications on the hydromechanical responses for a tunnel than for a spherical cavity. In general, increasing the backfill stiffness attenuates the transient peak response and leads to faster dissipation towards the long term equilibrium value. Conversely, a higher deterioration rate of the lining support accentuates the transient peak response and leads to a slower dissipation rate. The solutions presented in this paper have made it possible to compare the relative importance of these different parameters.

The present work is a first step to investigate the long term hydromechanical responses of a creeping surrounding ground following the closing of an underground disposal facility. This paper focuses on the behaviour of tunnels which are backfilled without removing their lining support. The period of time required to restore the hydraulic balance around the tunnel is neglected compared to the one required to degrade the lining support, hence fully saturated conditions are assumed here. Unsaturated behavior during the transient period will also be investigated in parallel works, where one of the key-steps is the development of adequate constitutive models to account for suction effects (Pereira et al., 2005; Morvan et al., 2009).

\section{REFERENCES}

1) Andra (2005): Dossier 2005 - Andra research on the geological disposal of high-level long-lived radioactive waste - Results and perspectives, Report Series, p. 5. 
2) Coussy, O. (1991): Mécanique des Milieux Poreux, Editions Technip.

3) Coussy, O. (2004): Poromechanics, John Wiley \& Sons Ltd.

4) Dufour, N. (2008) : Modélisation analytique et numérique du comportement hydromécanique poro-élastique d'une cavité souterraine remblayée, $M S c$, Ecole Nationale des Travaux de l'Etat.

5) Findley, W. N., Lai, J. S. and Onaran, K. (1976): Creep Relaxation of Nonlinear Viscoelastic Materials, Dover Publications, Inc., New York, N.Y.

6) Gaombalet, J. (2004): Le gonflement des argiles et ses effets sur les ouvrages souterrains de stockage, PhD Thesis, Ecole Polytechnique, Palaiseau, France.

7) Morvan, M., Wong, H. and Branque, D. (2009): An unsaturated soil model with minimal number of parameters, Int. J. Num. Analy. Meth. Geomech (submitted).

8) Pereira, J.-M. (2005): Etude des couplages hydromécaniques et effets de non-saturation dans les géomatériaux, Application aux ouvrages souterrains, PhD Thesis, Ecole Nationale des Travaux Publics de l'Etat (ENTPE).

9) Pereira, J. M., Wong, H., Dubujet, P. and Dangla, P. (2005): Adaptation of existing behaviour models to unsaturated states, Int. J. Num. Analy. Meth. Geomech., 29(11), 1127-1155.

10) Phienwej, N. (1987): Squeezing ground response of sheared shale of Steelwater Tunnel, PhD Thesis, Univ. of Illinois at Urbana-Champaign, Urbana, Ill.

11) Phienwej, N., Thakur, P. K. and Cording, E. J. (2007): Timedependent response of tunnels considering creep effect, Int. J. Geomechanics, 7(4), 296-306.

12) Semple, R. M. (1973): Effect of time dependent properties of altered rocks on tunnel support requirements, $P h D$ Thesis, Univ of Illinois at Urbana-Champaign, Urbana, Ill.

13) Stehfest, H. (1970): Algorithm 368, Commun. Assoc. Comput. Mach., 13(1), 47-49.

14) Talbot, A. (1979): The accurate inversion of Laplace transforms, $J$. Inst. Maths. Applics., 23, 97-120.

15) Terzaghi, K. (1946): Rock defects and loads on tunnel supports, Rock Tunnelling with Steel Supports (eds. by Proctor and White), Commercial Shearing and Stamping Co., Youngstown, Ohio.

16) Subrin, D. and Wong, H. (2002): Tunnel face stability in frictional material: a new 3D failure mechanism, Comptes Rendus Mecanique, 330(7), 513-519.

17) Wong, H., Morvan, M., Deleruyelle, F. and Leo, C. J. (2008a) Analytical study of mine closure behaviour in a poro-visco-elastic medium, Int. J. for Numerical and Analytical Methods in Geomechanics, 32(14), 1737-1761.

18) Wong, H., Morvan, M., Deleruyelle, F. and Leo, C. J. (2008b) Analytical study of mine closure behaviour in a poro-elastic medium, Computers and Geotechnics, 35(5), 645-654.

\section{APPENDIX A: RADIAL STRESS AT INTERIOR OF TUNNEL-LINER INTERFACE}

It is assumed that after closure, the tunnel is backfilled with a homogeneous poroelastic material and quickly becomes saturated. The initial effective stress in the backfill is supposed to be null. It is reasonable also to assume that changes in the density of the backfill are negligible relative to the changes in stresses, then, from consideration of equilibrium we write:

$$
\operatorname{div}\left(\sigma-\sigma^{0}\right)=0
$$

Noting that $\sigma^{0}=-p_{0} \mathbf{I}$, where $\mathbf{I}$ is the identity tensor, Eq. (A1) gives,

$$
\left(K_{\mathrm{R}}-\frac{2}{3} \mu_{\mathrm{R}}\right) \operatorname{div}(\epsilon \mathbf{I})-\operatorname{div}\left\{\left(p-p_{0}\right) \mathbf{I}\right\}+2 \mu_{\mathrm{R}} \operatorname{div}(\boldsymbol{\varepsilon})=0
$$

where $K_{\mathrm{R}}, \mu_{\mathrm{R}}$ are the bulk and shear moduli of the back- fill. Further manipulations give,

$$
\operatorname{grad}\left\{\left(K_{\mathrm{R}}+\frac{4}{3} \mu_{\mathrm{R}}\right) \epsilon-\delta p\right\}=0
$$

We shall assume that the permeability of the backfill is very high compared to the one of the surrounding medium so that $\operatorname{grad}(\delta p)=\mathbf{0}$ can reasonably be assumed for the backfill. In that case, it follows from Eq. (A3) that,

$$
\operatorname{grad}(\epsilon)=\mathbf{0}
$$

For a plane strain tunnel, since $\bar{\epsilon}=(\partial \bar{u} / \partial r)+(\bar{u} / r)$, Eq. (A4) leads to,

$$
\frac{\partial u}{\partial r}+\frac{u}{r}=2 k(t)
$$

where $k(t)$ is an arbitrary function of time. Integrating Eq. (A5) with respect to $r$ and appropriate boundary conditions, we get:

$$
u(r, t)=\frac{u\left(a^{-}, t\right)}{a} r ; \quad \epsilon\left(a^{-}, t\right)=\frac{2 u\left(a^{-}, t\right)}{a}
$$

Then using the relation

$$
\sigma\left(a^{-}, t\right)=K_{\mathrm{R}} \frac{2 u\left(a^{-}, t\right)}{a} \mathbf{I}-p(a, t) \mathbf{I}+2 \mu_{\mathrm{R}} \mathbf{e},
$$

where $\mathbf{e}$ is the deviatoric strain tensor and $r=a^{-}$indicates the radial distance on the interior of the lining support, gives radial stress at the interior of the tunnel-liner as:

$$
\sigma_{\mathrm{rr}}\left(a^{-}, t\right)=\left(2 K_{\mathrm{R}}+\frac{2}{3} \mu_{\mathrm{R}}\right) \frac{u(a, t)}{a}-p(a, t)
$$

\section{APPENDIX B: RADIAL STRESS AT EXTERIOR OF TUNNEL-LINER INTERFACE}

To determine the radial stress at the exterior of the tunnel-liner interface we revert to the relations:

$$
\begin{aligned}
& \bar{\sigma}_{\mathrm{rr}}=\bar{\sigma}+\bar{s}_{\mathrm{rr}} ; \quad \bar{\sigma}-\left(-\frac{\Sigma_{0}}{s}\right)=s \bar{K} \bar{\epsilon}-\delta \bar{p} ; \\
& \bar{S}_{\mathrm{rr}}=2 s \bar{\mu}\left(\frac{2 \bar{\epsilon}}{3}-\frac{\bar{u}}{r}\right)
\end{aligned}
$$

giving,

$$
\begin{aligned}
\bar{\sigma}_{\mathrm{rr}}\left(a^{+}, t\right)= & -\frac{\Sigma_{0}}{s}+s\left(\bar{K}+\frac{4}{3} \bar{\mu}\right) \bar{\epsilon}(a, s) \\
& -\delta \bar{p}(a, s)-2 s \bar{\mu} \frac{\bar{u}(a, s)}{a}
\end{aligned}
$$

However, from Eq. (34) we get,

$$
\delta \bar{p}=A K_{0}(q r) ;\left.\quad \frac{\partial(\delta \bar{p})}{\partial r}\right|_{\mathrm{r}=\mathrm{a}}=-A q K_{1}\left(q a^{+}\right)
$$

and from Eq. (30) we further obtain:

$$
-A \lambda_{\mathrm{h}} q K_{1}(q a)=s \bar{u}(a, s)
$$

so that, 


$$
A=-\frac{s}{\lambda_{\mathrm{h}} q K_{1}(q a)} \bar{u}(a, s)
$$

Backsubstituting into Eqs. (B2) and (B3), then using Eq.

we arrive at the expression:

(33) with the results of APPENDIX A and noting from where Eq. (21) that:

$$
\bar{\epsilon}\left(a^{+}, t\right)=\frac{\partial \bar{u}(a, s)}{\partial t}+\frac{\bar{u}(a, s)}{a}=\frac{\delta \bar{p}\left(a^{+}, s\right)}{s\left(\bar{K}+\frac{4 \bar{\mu}}{3}\right)}
$$

$$
\bar{u}(a, s)=\frac{-\kappa a\left(\Sigma_{0}-p_{0}\right)}{s(s+\kappa) \Omega(s)}
$$

$$
\Omega(s)=\left(2 K_{\mathrm{R}}+\frac{2}{3} \mu_{\mathrm{R}}+2 s \bar{\mu}\right)+\frac{a s}{\lambda_{\mathrm{h}} q} \frac{K_{0}(q a)}{K_{1}(q a)}
$$

